\title{
CORRESPONDENCE
}

\section{Serum procalcitonin level: clinical significance}

\section{To the Editors:}

The study by UGAJIN et al. [1] nicely evaluates the role of procalcitonin in differentiating community-acquired pneumonia (CAP) from pulmonary tuberculosis (PTB). They have further highlighted its prognostic value in PTB. Few aspects regarding this promising biomarker need to be discussed to understand its clinical significance.

Procalcitonin, a propeptide of calcitonin, is an important host response biomarker indicative of infection. In particular, it aids in assessing the severity of infection and its prognosis $[2,3]$. Using this severity marker to differentiate two infectious diseases seems unjustified. Both the diseases can have varying degrees of severity and may also be confounded by factors such as immunocompromised status and presence of comorbidities, such as post surgery [4], trauma [5], acute exacerbations of chronic obstructive pulmonary disease and acute coronary syndrome, which can alter procalcitonin levels. Clinical presentations, pattern of radiological involvement and sputum examination still remain the gold standard, as well as cost-effective measures to differentiate PTB and CAP.

Serum procalcitonin, although an important prognostic marker, may not be useful in the presence of the aforementioned comorbidities. Extensive radiological involvement, multiorgan infection, high sputum positivity and drug resistance are more reliable markers for severity of tuberculosis and poor prognosis. Serum procalcitonin measurement should be performed in selected cases of PTB where its positivity should alert the clinician to search for tubercular foci in other organs of the body.

\section{Aggarwal*, P.R. Mohapatra" and P. Aggarwal}

*Pulmonary and Critical Care, Fortis-Escorts Hospital, "Department of Pathology, Sri Guru Ram Das Institute of Medical Sciences and Research, Amritsar, and "Department of Pulmonary Medicine, Government Medical College and Hospital, Chandigarh, India.

Correspondence: D. Aggarwal, House no - 11-E, Gali No-5, Hukam Singh Road, Amritsar, Punjab, India. E-mail: aggarwaldr@ yahoo.co.in

Statement of Interest: None declared.

\section{REFERENCES}

1 Ugajin M, Miwa S, Shirai M, et al. Usefulness of serum procalcitonin levels in pulmonary tuberculosis. Eur Respir J 2011; 37: 371-375.

2 Delevaux I, Andre M, Colombier M, et al. Can procalcitonin measurement help in differentiating between bacterial infection and other kinds of inflammatory processes? Ann Rheum Dis 2003; 62: 337-340.

3 Christ-Crain M, Opal SM. Clinical review: the role of biomarkers in the diagnosis and management of community-acquired pneumonia. Crit Care 2010; 14: 203.

4 Meisner M, Tschaikowsky K, Hutzler A, et al. Postoperative plasma concentrations of procalcitonin after different types of surgery. Intensive Care Med 1998; 24: 680-684.

5 Wanner GA, Keel M, Steckholzer U, et al. Relationship between procalcitonin plasma levels and severity of injury, sepsis, organ failure, and mortality in injured patients. Crit Care Med 2000; 28: 950-957.

DOI: 10.1183/09031936.00132310

From the author:

We are grateful to D. Aggarwal and co-workers for their comments on our recent article in the European Respiratory Journal [1]. As they indicated, the serum procalcitonin (PCT) level could be altered under various conditions and we agree that clinical manifestations, sputum examination and radiological findings are the gold standard to differentiate pulmonary tuberculosis (PTB) from community-acquired pneumonia. Our data showed that serum PCT is not chronically elevated in PTB patients and that it is a poor prognostic marker when the level is above normal. Therefore, we also consider that serum PCT should be measured in selected cases in terms of its practical cost-benefit performance.

\section{S. Miwa}

Correspondence: S. Miwa, Dept of Respiratory Medicine, Tenryu Hospital, National Hospital Organization, 4201-2 Oro, Hamakitaku, Hamamatsu, 434-8511, Japan. E-mail: hirosei@za. tnc.ne.jp

Statement of Interest: None declared.

\section{REFERENCES}

1 Ugajin M, Miwa S, Shirai M, et al. Usefulness of serum procalcitonin levels in pulmonary tuberculosis. Eur Respir J 2011; 37: 371-375.

DOI: $10.1183 / 09031936.00144410$ 